\title{
ALMOST-COMPLEX SUBSTRUCTURES ON THE SPHERE
}

\author{
I. DIBAG
}

Abstract. The paper solves completely the existence problem of almostcomplex substructures on spheres.

1. Introduction. An almost-complex $s$-substructure on an orientable $n$-manifold $M$ is defined to be a $2 s$-dimensional sub-bundle of the tangent-bundle $T(M)$ that admits an almost-complex structure, i.e. a (continuous) structure map $J, J^{2}=-1$. Assuming a metric on $T(M)$, we call an almost-complex substructure $J$ normalized iff $J$ is also orthogonal on the sub-bundle of its definition. If $J$ is an almost-complex $s$-substructure, then the orthogonal-component in its polar-decomposition (into an orthogonal and a positive-definite symmetric transformation) defines a normalized almost-complex $s$-substructure. Analogously, if $w$ is a 2 -form on $M$ of constant rank $2 s$, the union of the $2 s$-planes on which $w$ is of maximal rank forms a sub-bundle $S_{w}$ of $T(M)$, and the orthogonal-component of $w$ (regarded as a nonsingular, skew-adjoint transformation on $S_{w}$ ) defines a normalized almost-complex $s$-substructure (e.g. refer to [3]). We thus have: Existence of an almost-complex $s$-substructure $\Leftrightarrow$ Existence of a normalized almost-complex $s$-substructure $\Leftrightarrow$ Existence of a 2 -form on $M$ of constant rank $2 s$.

The purpose of this paper is to solve completely the existence problem of almost-complex substructures on spheres. In particular, we shall prove the following Theorem.

Let $c_{k}=$ dimension of the irreducible (ungraded) representation module for the Clifford-Algebra $C_{k}$, i.e. $c_{k}=2^{e_{k}}$ where $e_{k}=$ number of integers $t, 0<t$ $\leqslant k, t=0,1,2,4(\bmod 8)$.

Let $M_{k}$ be the Atiyah-Todd number defined in [2], i.e. if for an integer $n$ and a prime $p, v_{p}(n)$ denotes the exponent of $p$ in the prime-factorization of $n$, then

$$
v_{p}\left(M_{k}\right)= \begin{cases}\sup \left(r+v_{p}(r)\right), \quad 1 \leqslant r \leqslant[(k-1) /(p-1)] & \text { if } p \leqslant k \\ 0 & \text { if } p>k .\end{cases}
$$

Let $L_{k}$ be the 2-component of $M_{k}$, i.e.

$$
L_{k}=2^{v_{2}\left(M_{k}\right)} \text { where } v_{2}\left(M_{k}\right)=\sup \left(r+v_{2}(r)\right), 1 \leqslant r \leqslant k-1 .
$$

Received by the editors July 21, 1975 and, in revised form, March 22, 1976.

AMS (MOS) subject classifications (1970). Primary 53C15, 55G40; Secondary 53C65.

Key words and phrases. Almost-complex, contact, symplectic, almost product structures, etc., sectioning fiber spaces and bundles, integral geometry, differential forms and currents. 
THEOREM. I. The only almost-complex substructures on even-spheres are the almost-complex structures on $S^{2}$ and $S^{6}$.

II. (i) For $s<(2 n-1) / 4 ; S^{2 n-1}$ admits an almost-complex s-substructure iff $c_{2 s} / 2 n$. (ii) For $s>(2 n-1) / 4 ; S^{2 n-1}$ admits an almost-complex $s$-substructure iff $L_{n-s} / n$.

I, II(i) and II(ii) for $n$ odd and $n=2,4$ are either known, or else, easy consequences of known material and will be quickly dealt with in $\S 2$. The main task of the paper will be to prove II(ii) for $n$ even, $n>4$.

$\S 3$ will reduce the problem to one which can be effectively tackled; and $\S 4$ will further reduce it to the order of the obstruction $w_{n, n-s}$ of the complex Stiefel-Fibering,

$$
W_{n, n-s} \stackrel{W_{n-1, n-s-1}}{\rightarrow} S^{2 n-1}
$$

The Theorem will then follow from the case $p=2$ of Theorem I of [4].

2. Elimination of certain special cases. The main tools for this section are the following:

LEMMA 2.1. If $2 k \leqslant n$, then $S^{n}$ admits a field of $k$-planes iff it admits a field of $k$-frames.

Proof. Refer to [5, p. 144, Theorem 27.16].

COROllary. Let $S^{n}$ admit an s-substructure.

(i) If $s \leqslant n / 4$, it admits a field of $2 s$-frames.(ii) If $s>n / 4$, it admits a field of $(n-2 s)$-frames.

LEMMA 2.2. $S^{2 n-1}$ admits an $(n-1)$-substructure.

Proof. The canonical complex-structure $i$ on $R^{2 n}$ defines a 1 -frame, $x \mapsto i x, x \in S^{2 n-1}$, on $S^{2 n-1}$ whose orthogonal-complement in $T\left(S^{2 n-1}\right)$ is preserved under $i$ and thus defines an $(n-1)$-substructure on $S^{2 n-1}$.

We now proceed to prove the easy part of the Theorem.

Proof OF I. It is well known that $S^{2}$ and $S^{6}$ are the only even dimensional spheres that admit almost-complex structures. If $S^{2 n}$ admits a proper substructure (i.e. $s<n$ ), then by the Corollary to Lemma 2.1 it admits at least a 1-frame, which is impossible.

Proof OF II(i). It is a consequence of the Corollary to Lemma 2.1 and the result of Adams (e.g. refer to [1]) that $S^{2 n-1}$ admits a field of 2 s-frames iff $c_{2 s} / 2 n$.

PrOOF OF II(ii) FOR $n$ ODD. Suppose $S^{2 n-1}$ admits an $s$-substructure for $n$ odd and $s>(2 n-1) / 4$. By the Corollary to Lemma 2.1, it admits a field of $(2 n-2 s-1)$-frames; and $2 n-2 s-1 \leqslant 1$, i.e. $s \geqslant n-1$ by [1]. Applying Lemma 2.2 yields $s=n-1$. Since $L_{1}=1, L_{2}=2$, this is, precisely, what the Theorem states.

ProOF OF II(ii) FOR $n=2,4$. Obvious, since $S^{2 n-1}$ is parallelisable. 


\section{Reduction of the problem.}

3.1. Homotopy exact sequence of $S O(2 n-1) \rightarrow S O(2 n) \rightarrow^{p} S^{2 n-1}$. Consider the exact sequence

$$
\begin{array}{cccc}
\pi_{2 n-1} S O(2 n) \stackrel{p_{\#}}{\rightarrow} \pi_{2 n-1} S^{2 n-1} & \rightarrow \pi_{2 n-2} S O(2 n-1) & \rightarrow \pi_{2 n-2} S O(2 n) \\
\| & & \pi_{2 n-2} S O \\
& & \| \\
& \text { gen. } \iota_{2 n-1} & 0 n \text { even }
\end{array}
$$

$0 n$ even

By Theorem 23.4 of [5], $2 \iota_{2 n-1} \in \operatorname{Im} p_{\#}$. Hence $\pi_{2 n-2} S O(2 n-1)=0$ or $Z_{2}$ generated by $\partial\left(t_{2 n-1}\right) \cdot \pi_{2 n-1} S O(2 n-1)=0$ iff $p$ admits a cross-section iff $S^{2 n-1}$ is parallelisable. Thus, for $n$ even, $n>4$,

$$
\pi_{2 n-2} S O(2 n-1)=Z_{2} \text {. }
$$

As remarked in the introduction, $S^{2 n-1}$ admits an $s$-substructure iff it admits a normalized $s$-substructure. A normalized $s$-substructure on $S^{2 n-1}$ is, simply, a reduction of the structure-group of $T\left(S^{2 n-1}\right)$ from $S O(2 n-1)$ to $U(s) \times S O(2 n-2 s-1)$; and is, thus, a cross-section to the fibration:

(i) $S O(2 n) / U(s) \times S O(2 n-2 s-1) \stackrel{\operatorname{SO}(2 n-1) / U(s) \times S O(2 n-2 s-1)}{\rightarrow} S^{2 n-1}$.

Define $\Gamma_{m, s}=S O(m) / U(s)$ for $2 s \leqslant m$. We have a fibration:

$$
\Gamma_{2 n, s}=S O(2 n) / U(s) \stackrel{\Gamma_{2 n-1, s}=S O(2 n-1) / U(s)}{\rightarrow} S^{2 n-1} .
$$

Let $V_{n, k}=S O(n) / S O(n-k)$ be the Stiefel-Manifold of orthonormal $k$-frames in $R^{n}$. We have a third fibration:

$$
V_{2 n, 2 s+1} \stackrel{V_{2 n-1,2 s}}{\rightarrow} S^{2 n-1}
$$

The following proposition will reduce the problem to one which can be effectively tackled.

Proposition 3.2. For $n$ even, $n>4, s>(2 n-1) / 4$, there exists an $s$-substructure on $S^{2 n-1}$ iff the fibration (ii) admits a cross-section.

Proof. Let

$$
j: U(s) \subset S O(2 n-1) \text { and } k: S O(2 n-2 s-1) \subset S O(2 n-1)
$$

be the inclusions. Let

$$
\begin{aligned}
& \pi_{2 n-2} U(s) \oplus \pi_{2 n-2} S O(2 n-2 s-1) \stackrel{\left(j_{\#}, k_{\#}\right)}{\rightarrow} \pi_{2 n-2} S O(2 n-1) \\
& \stackrel{\gamma_{\#}}{\rightarrow} \pi_{2 n-2} S O(2 n-1) / U(s) \times S O(2 n-2 s-1), \\
& \pi_{2 n-2} U(s) \stackrel{j_{\#}}{\rightarrow} \pi_{2 n-2} S O(2 n-1) \stackrel{\beta_{\#}}{\rightarrow} \pi_{2 n-2} \Gamma_{2 n-1, s}, \\
& \pi_{2 n-2} S O(2 n-2 s-1) \stackrel{k_{\#}}{\rightarrow} \pi_{2 n-2} S O(2 n-1) \stackrel{v_{\#}}{\rightarrow} \pi_{2 n-2} V_{2 n-1,2 s}
\end{aligned}
$$

be the exact homotopy sequences of the projections: 


$$
\begin{aligned}
& \gamma: S O(2 n-1) \rightarrow S O(2 n-1) / U(s) \times S O(2 n-2 s-1), \\
& \beta: S O(2 n-1) \rightarrow \Gamma_{2 n-1 . s} \text { and } \quad v: S O(2 n-1) \rightarrow V_{2 n-1,2 s} .
\end{aligned}
$$

Let $a_{n} \in \pi_{2 n-2} S O(2 n-1)=Z_{2}$ be the generator and also the obstruction to cross-sectioning

$$
p: S O(2 n) \stackrel{S O(2 n-1)}{\rightarrow} S^{2 n-1} .
$$

Then the obstructions to cross-sectioning (i), (ii), and (iii) are $\gamma_{\#}\left(a_{n}\right), \beta_{\#}\left(a_{n}\right)$ and $v_{\#}\left(a_{n}\right)$, respectively. The work of Adams [1] shows that $S^{2 n-1}$ does not admit a field of $2 s$-frames for $n>4$ and $s>(2 n-1) / 4$. Thus, (iii) cannot be sectioned and hence $v_{\#}\left(a_{n}\right) \neq 0$. Since $\pi_{2 n-2} S O(2 n-1)=Z_{2}, v_{\#}$ is a monomorphism. Thus, $k_{\#}=0$ by exactness. Hence (i) admits a cross-section iff $\gamma_{\#}\left(a_{n}\right)=0$, iff $a_{n} \in \operatorname{Image}\left(j_{\#}, k_{\#}\right)$, iff $a_{n} \in \operatorname{Image} j_{\#}$, iff $\beta_{\#}\left(a_{n}\right)=0$, iff (ii) admits a cross-section. Q.E.D.

Cross-sections to (ii) are normalized $s$-substructures on $S^{2 n-1}$ whose subbundles of definition have "trivial" orthogonal-complements in $T\left(S^{2 n-1}\right)$. Such $s$-substructures will be called complement-split. We now slightly generalize Proposition 3.2.

Proposition 3.3. For $s>(2 n-1) / 4, S^{2 n-1}$ admits an $s$-substructure iff it admits a complement-split $s$-substructure.

Proof. For $n$ odd, the only possible $s>(2 n-1) / 4$ such that $S^{2 n-1}$ admits an $s$-substructure is $s=n-1$; and a complement-split $(n-1)$ substructure is constructed on $S^{2 n-1}$ in Lemma 2.2. The proof for $n$ even, $n>4$ is furnished by Proposition 3.2, and is obvious for $n=2$, 4. Q.E.D.

4. The boundary-operator. Since $U(n) \cdot S O(2 n-1)=S O(2 n)$ and $U(n)$ $\cap S O(2 n-1)=U(n-1)$,

$$
\Gamma_{n}=S O(2 n) / U(n)=S O(2 n-1) / U(n-1) .
$$

Let

$$
\begin{aligned}
& u: S O(2 n-1) \stackrel{U(n-1)}{\rightarrow} \Gamma_{n} \rightarrow \pi_{2 n-1} S O(2 n-1) \stackrel{u_{\#}}{\rightarrow} \pi_{2 n-1} \Gamma_{n} \\
& \stackrel{\partial_{u}}{\rightarrow} \pi_{2 n-2} U(n-1) \rightarrow \ldots
\end{aligned}
$$

be the exact homotopy sequence of $u$. We have two more fibrations:

$$
\begin{aligned}
W_{n, n-s} & =U(n) / U(s) \stackrel{W_{n-1, n-s-1}=U(n-1) / U(s)}{\rightarrow} S^{2 n-1} . \\
\Gamma_{2 n-1, s} & =S O(2 n-1) / U(s)^{W_{n-1, n-s-1}=U(n-1) / U(s)} \rightarrow \Gamma_{n} \\
& =S O(2 n-1) / U(n-1) .
\end{aligned}
$$

Let $w_{n, n-s} \in \pi_{2 n-2} W_{n-1, n-s-1}$ denote the obstruction to cross-sectioning fibration (iv). The purpose of this section is to determine the image of $\partial_{u}$, and hence further reduce the problem to an equivalent statement about the order of $w_{n, n-s}$. 
Let $H, K$ be closed subgroups of a compact topological group $G$ with $H K=K H$, so that $H K$ is a compact subgroup of $G$. Let

$$
\begin{gathered}
p^{1}: H K / H \cap K \stackrel{K / H \cap K}{\rightarrow} H K / K=H / H \cap K, \\
p^{2}: H K / H \cap K \stackrel{H / H \cap K}{\rightarrow} H K / H=K H / H=K / H \cap K
\end{gathered}
$$

be the projections. Let $\partial, \partial^{H}, \partial^{K}$ be the boundary-operators of the fibrations: $t: H K \stackrel{H}{H} \rightarrow^{\rightarrow} H K / H \cap K, \quad t^{1}: H \stackrel{H \cap K}{\rightarrow} H / H \cap K, \quad t^{2}: K \stackrel{H \cap K}{\rightarrow} K / H \cap K$ respectively. We state the following

Proposition 4.1. (i) $\pi_{q} H K / H \cap K=\pi_{q} H / H \cap K \oplus \pi_{q} K / H \cap K$ where $p_{\#}^{1}$ and $p_{\#}^{2}$ define the projections onto the factors. (ii) Relative to this decomposition, $\partial$ decomposes as a sum of operators:

Proof. Elementary exercise.

$$
\partial=\partial^{H} \oplus \partial^{K} .
$$

Let $q^{1}: H K \rightarrow^{K} H / H \cap K, q^{2}: H K \rightarrow^{H} K / H \cap K$ denote the projections, i.e. $q^{1}=p^{1} t$ and $q^{2}=p^{2} t$.

Corollary 4.1. The diagram below is ANTI-commutative.

$$
\begin{array}{ccc}
\pi_{q} H K & \stackrel{q_{\#}^{1}}{\rightarrow} & \pi_{q} H / H \cap K \\
\downarrow q_{\#}^{2} & & \downarrow \partial^{H} \\
\pi_{q} K / H \cap K & \stackrel{\partial^{K}}{\rightarrow} & \pi_{q-1} H \cap K
\end{array}
$$

Proof. Let $x \in \pi_{q} H K$. By Proposition $4.10=\partial t_{\#}(x)=\partial{ }^{H} p_{\#}^{1} t_{\#}(x)$ $+\partial{ }^{K} p_{\#}^{2} t_{\#}(x)=\partial^{H} q_{\#}^{1}(x)+\partial{ }^{K} q_{\#}^{2}(x)$ i.e. $\partial^{H} q_{\#}^{1}=-\partial^{K} q_{\#}^{2}$. Q.E.D.

PROPOSITION 4.2. Let $u: S O(2 n-1) \rightarrow^{U(n-1)} \Gamma_{n}$ and $\partial_{u}: \pi_{2 n-1} \Gamma_{n} \rightarrow$ $\pi_{2 n-2} U(n-1)$ be the boundary-homomorphism in the homotopy exact sequence of $u$. Then Image $\partial_{u}$ is the subgroup generated by $2 w_{n, n}$, where $w_{n, n}$ is the obstruction to a cross-section of $U(n) \rightarrow^{U(n-1)} S^{2 n-1}$.

Proof. Consider the following diagram.

$$
\begin{array}{lllll}
\pi_{2 n-1} S O(2 n) & \stackrel{r_{\#}}{\rightarrow} & \pi_{2 n-1} \Gamma_{n} & \stackrel{\partial_{r}}{\rightarrow} & \pi_{2 n-2} U(n)=0 \\
\downarrow p_{\#} & & \downarrow \partial_{u} & \\
\pi_{2 n-1} S^{2 n-1} & \stackrel{\partial_{q}}{\rightarrow} & \pi_{2 n-2} U(n-1) &
\end{array}
$$

The row is part of the homotopy exact sequence of $r: S O(2 n) \rightarrow^{U(n)} \Gamma_{n}$. The square is anticommutative, and is obtained from Corollary 4.1 by taking

$$
\begin{aligned}
& H=S O(2 n-1), \quad K=U(n), \\
& G=H K=K H=S O(2 n), \quad H \cap K=U(n-1) .
\end{aligned}
$$

By exactness, $r_{\#}$ is an epimorphism. Hence

$$
\operatorname{Im} \partial_{u}=\operatorname{Im}\left(\partial_{u} r_{\#}\right)=\operatorname{Im}\left(\partial_{q} p_{\#}\right) ;
$$


now use 3.1 and the fact that $w_{n, n}=\partial_{q} \iota_{2 n-1}$. Q.E.D.

Proposition 4.3. For $n$ even, $n>4$, the fibration (ii) admits a cross-section iff $w_{n, n-s}$ is of odd order.

Proof. Consider the following commutative diagram, where the bottom row is part of the homotopy exact sequence of the fibration (v).

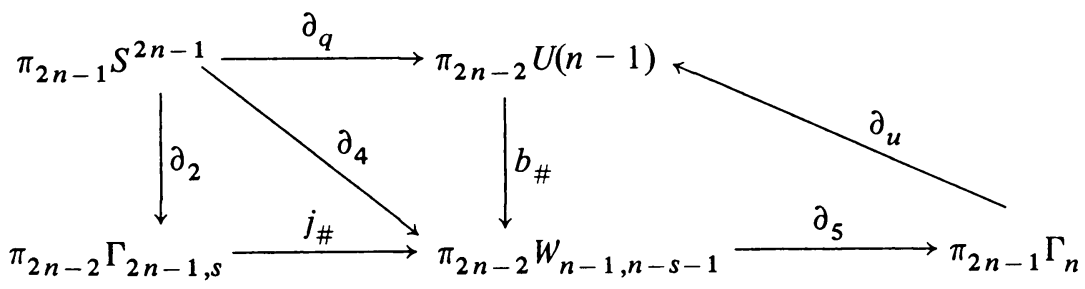

(ii) admits a cross-section iff $\partial_{2}=0$ iff $j_{\#} \partial_{4}=0$ iff $w_{n, n-s} \in \operatorname{Im} \partial_{5}$ and $\operatorname{Im} \partial_{5}=b_{\#}\left(\operatorname{Im} \partial_{u}\right)$. By Proposition 4.2 above and the fact that $b_{\#}\left(w_{n, n}\right)=$ $w_{n, n-s}, b_{\#}\left(\operatorname{Im} \partial_{u}\right)$ is the subgroup generated by $2 w_{n, n-s}$. Hence $w_{n, n-s} \in \operatorname{Im} \partial_{5}$ iff it is of odd order. Q.E.D.

Finally, let $p$ be a prime. Theorem I of [4] states that the order of the obstruction $w_{n, k}$ is prime to $p$ iff $n$ is divisible by the $p$-primary-component of $M_{k}$.

Thus, the proof of II(ii) for $n$ even, $n>4$, of the Main Theorem follows from Propositions 3.2 and 4.3 and the case $p=2$ of Theorem I of [4].

The proof of the Main Theorem for $s>(2 n-1) / 4$ is "existential", and, unfortunately, does not give explicit constructions, except for the $(n-1)$ substructure on $S^{2 n-1}$ defined in Lemma 2.2, using the complex-structure on $R^{2 n}$. Analogously, for $n$ even, one can construct an $(n-2)$-substructure on $S^{2 n-1}$ using the quaternionic structure on $R^{2 n}$; and these are the only constructions known to the author. It is hoped that one will explicitly construct $s$-substructures on $S^{2 n-1}$ for $s>(2 n-1) / 4$ whenever possible, i.e. when $L_{n-s} / n$; and this will throw more light onto the problem.

\section{REFERENCES}

1. J. F. Adams, Vector fields on spheres, Ann. of Math. (2) 75 (1962), 603-632. MR 25 \#2614.

2. M. F. Atiyah and J. A. Todd, On complex Stiefel manifolds, Proc. Cambridge Philos. Soc. 56 (1960), 342-353. MR 24 \# A2392.

3. I. Dibag, Decomposition in the large of two-forms of constant-rank, Ann. Inst. Fourier (Grenoble) 24 (3) (1974), 317-335.

4. F. Sigrist, Sur les nombres de James des variétés de Stiefel complexes, Illinois J. Math. 13 (1969), 198-201. MR 40 \#2078.

5. N. E. Steenrod, The topology of fibre bundles, Princeton Univ. Press, Princeton, N. J., 1951. MR 12, 522 .

Department of Mathematics, Middle-East Technical University, Ankara, Turkey 